\title{
Funciones isoelásticas de utilidad, rejillas trinomiales, volatilidad y aversión al riesgo cambiante: un modelo de valuación con opciones reales de empresas de base tecnológica
}

\author{
Gastón S. Milanesi
}

Recibido: 20 de mayo de 2020

Aprobado: 20 de julio de 2020

Milanesi, G. (2020). Funciones isoelásticas de utilidad, rejillas trinomiales, volatilidad y aversión al riesgo cambiante: un modelo de valuación con opciones reales de empresas de base tecnológica. Revista Activos, 18(2), 25-54. https://doi.org/10.15332/25005278/6258

\section{Clasificación JEL: G17, G31}

* Doctor en Ciencias de la Administración; profesor titular a tiempo completo y director del doctorado en Ciencias de la Administración de la Universidad Nacional del Sur; profesor en la Licenciatura en Organización Industrial de la Universidad Tecnológica Nacional; investigador en el Centro de Estudios en Administración de la Universidad Nacional del Sur, en el Centro de Estudios para Análisis Financiero de la Facultad de Ciencias Económicas de la Universidad de Buenos Aires, y, en el Departamento de Licenciatura en Organización Industrial en la Facultad Regional Bahía Blanca de la Universidad Tecnológica Nacional. Correo electrónico: milanesi@uns.edu.ar ORCID: https://orcid.org/0000-0003-1759-6448 


\section{Resumen}

La valoración de inversiones en I\&D, intangibles y empresas de base tecnológica (ЕВт) requiere modelos que valoren la flexibilidad estratégica, la volatilidad cambiante según el ciclo de vida y los riesgos sin activos financieros gemelos. En mercados incompletos e imperfectos, los riesgos deben valorarse con funciones de utilidad del inversor. Por lo tanto, se propone un modelo de valuación de opciones reales con rejillas trinomiales, volatilidad cambiante, funciones isoelásticas de utilidad y aversión al riesgo variable. Se ilustra su funcionamiento mediante un análisis de caso y un estudio de sensibilidad sobre valuación de ЕвT. Los resultados obtenidos demuestran su versatilidad; además, el modelo captura riesgos privados y la flexibilidad estratégica como herramienta de valuación para este tipo de inversiones dentro de mercados ambiguos, imperfectos e incompletos.

Palabras clave: funciones isoelásticas, aversión al riesgo, volatilidad cambiante, rejillas trinomiales, empresas de base tecnológica. 


\title{
Isoelastic utility functions, trinomial lattices, volatility and changing risk aversion: a valuation model with real options of technology-based firms
}

\begin{abstract}
The valuation of investments in R\&D, intangibles and technological-based firms $(\mathrm{TBF})$ requires models that value strategic flexibility, changing volatility according to life cycle and risks without twin financial assets. In incomplete and imperfect markets, risks must be priced with investor's utility function. Therefore, a real option valuation model with trinomial lattice, changing volatility, isoelastic utility functions and varying risk aversion is proposed. Its performance is shown through a case analysis and a sensitivity study on TBF valuation. The results obtained demonstrate its versatility; moreover, the model captures private risk and strategic flexibility as a valuation tool, for this type of investments in ambiguous, imperfect and incomplete markets.
\end{abstract}

Keywords: isoelastic functions, risk aversion, changing volatility, trinomial lattice, technology-based firms. 


\section{Introducción}

Un proceso de valuación implica una actividad intelectual que tome conocimiento del activo real (objeto de valuación) con el fin de generar información cualitativa y cuantitativa para formar juicios de valor en relación con el valor del activo. El resultado de la valuación es subjetivo y refleja expectativas cuantitativas relativas a la corriente de beneficios futuros del activo. En la negociación, adquirente y vendedor, parten de sus valuaciones y acuerdan un precio de transferencia que refleja las expectativas del valor del activo. Entendido como tal, el precio contiene expectativas de valor, vinculadas al espacio temporal donde se perfeccionó la transacción. En el caso de los activos financieros, con mercados desarrollados, perfectos y completos, el precio coincide y se ajusta rápidamente a las expectativas de los agentes, es decir al valor (Copeland y Antikarov, 2001; Milanesi, 2019; y Wilmott, 2009). En el caso de transacciones con activos reales, la velocidad de convergencia entre el valor y el precio presenta menor celeridad, profundizándose en la medida en que el mercado sea incompleto y no sea perfecto, ni desarrollado.

Si a estas circunstancias, se añade el desafío de valuar empresas de base tecnológica, estrategias de innovación y desarrollo o intangibles, la divergencia entre valor y precio crece exponencialmente, ya que el último es inexistente. El agente debe formarse un juicio de valor en un contexto de mercados emergentes y para una inversión de base intangible, caracterizada por: (a) ambigüedad o inexistencia de información comparable o de mercado sobre los riesgos asociados a los flujos de la nueva inversión; (b) complejidad de la inversión, dada por sus múltiples fuentes de opcionalidad, volatilidades cambiantes a lo largo de su ciclo de vida y sesgos en su valor; y (c) preferencias o grado de aversión al riesgo del inversor, quien por restricciones del medio, no puede replicar riesgos del proyecto con carteras de cobertura, frente a mercados no completos. El modelo numérico de valuación debe brindar herramientas que permitan lidiar con los escollos enumerados. 
En ese orden de ideas, este trabajo propone un modelo numérico de valuación de empresas de bases tecnológicas, intangibles y estrategias en I\&D, entre otras, que considere los desafíos de valuación planteados. Por ello, se propone un modelo que utiliza rejillas trinomiales, con volatilidad cambiante según el estado de vida del proyecto, sesgos del valor y valoración con funciones exponenciales isoelásticas de utilidad, con grados cambiantes de aversión al riesgo. Dicho modelo tiene fundamentos y conjuga las propuestas contenidas en varios trabajos (Haahtela, 2011a, 2011b; Maya Ochoa y Pareja Vasseur, 2014; Milanesi 2018a, 2018b y 2019; Milanesi, Pesce y El Alabi, 2014; Pareja Vasseur y Cadavid, 2016; Pareja Vasseur y Baena, 2018).

Este trabajo presenta, primero, el modelo: a partir de las rejillas binomiales hasta las trinomiales, se incorpora la volatilidad cambiante y los sesgos en la proyección del valor correspondiente al proyecto. En segundo lugar, se incoporan las funciones isoleásticas, exponencias con coeficientes variables de aversión al riesgo. En la tercera sección, se analiza el caso de una empresa de base teconológica (ЕВТ) con opción de transferencia o expansión. El modelo es sometido a un análisis de sensibilidad bivariado relativo al sesgo y amplitud de movimientos en cada nodo de la rejilla. Después, se comparan los resultados obtenidos con el modelo binomial clásico, trinomial clásico, trinomial clásico con volatilidad cambiante, trinomial con volatilidad cambiante y sesgo. Finalmente, se realiza una conclusión sobre la ventajas del modelo numérico propuesto, puesto que su estructura permite incorporar los elementos que hacen a la complejidad del activo: flexibilidad estratégica, ambigüedad de datos, sesgo en su valor, riesgos cambiantes y sujetos no neutrales al riesgo.

\section{El modelo trinomial con volatilidad cambiante, desplazamiento y funciones isoelásticas de utilidad}

En esta sección se desarrollan los pilares del modelo numérico de valoración propuesto: desde los fundamentos en las rejillas binomiales hacia las rejillas trinomiales; la incorporación de volatilidad cambiante; el sesgo en el valor 
esperado del subyacente; y el uso de funciones isoelásticas exponenciales de utilidad.

\section{Rejillas binomiales}

Las rejillas son herramientas empleadas para proyectar el valor del activo subyacente y valorar opciones, presentando ventajas sobre los árboles de decisión debido a sus propiedades recombinantes de nodos intermedios (Smith, 2005) $)^{1}$. Se caracterizan por modelar en tiempo discreto el proceso estocástico continuo de la variable y utilizar el supuesto de valuación neutral al riesgo (Wilmott, 2009)2 . En el modelo binomial:

a. El precio del subyacente $S_{o}$ asciende a $S_{u}$, o desciende a $S_{d}$ en intervalos de tiempo $\Delta t$.

b. La probabilidad de transición correspondiente al movimiento ascendente es $p$, y su complemento para el descendente es 1-p.

Los parámetros del modelo $p, u, q \mathrm{y} d$ no pueden fijarse arbitrariamente. Los valores del primer y segundo momento estocástico son derivados del proceso estocástico geométrico/aritmético browniano (Copeland y Antikarov, 2001; Milanesi, 2014a). En el límite, los modelos binomiales convergen al modelo Black-Scholes (Black y Scholes, 1972 y 1973) de valoración

1 La propiedad de recombinación de las rejillas asegura que en el paso $\mathrm{N}$ existe $\mathrm{N}+$ 1 nodos finales y $n(n+1) / 2$ puntos de decisión mientras que en los árboles binomiales la cantidad de nodos finales asciende a $2^{n-1}$ y los puntos de decisión a $2^{n}-1$.

2 Se basa en la perfecta correlación entre los cambios en el valor de la opción y del activo subyacente. Una cartera compuesta por una posición larga (corta) en una opción y corta (larga) en el subyacente hace que el activo riesgoso cubra las fluctuaciones en el precio del derivado. El valor de la cartera crece al tipo sin riesgo y el número exacto de subyacente a vender (comprar) se conoce como "delta". En un mundo como el que plantea al modelo Black-Scholes, si se compran "delta" acciones empleando el modelo, entonces se cubre correctamente el riesgo eliminándolo por completo. 
de opciones en la medida que se cumpla con el teorema central del límite 3 . El sistema de ecuaciones que debe satisfacerse para la media y varianza es:

$$
\begin{aligned}
& E\left(\ln \left(\frac{V_{t}}{V_{0}}\right)\right)=p \cdot \ln (u)+(1-p) \ln (d)=u . \Delta t=e^{r \Delta t} \\
& \operatorname{Var}\left(\ln \left(\frac{V_{t}}{V_{0}}\right)\right)=p(1-p)\left[\ln \frac{u}{d}\right]^{2}=\sigma^{2} \cdot \Delta t=e^{\left(2 r+\sigma^{2}\right) \Delta t} \\
& p+p_{d}=1 ; 0<p<1
\end{aligned}
$$

Donde $\Delta t$ es igual a $T / n$; $T$ es el tiempo hasta el horizonte final de proyección y $n$ es el número de pasos. La otra restricción en las rejillas es la condición de recombinación,

$$
u . d=d \cdot u=m^{2}
$$

Existen tres ecuaciones y cuatro incógnitas $(p, u, d y q)$, siendo la cuarta ecuación impuesta de manera arbitraria (Wilmott, 2009). Es aquí donde la variedad y gama de posibles formulaciones de rejillas binomiales varía en función de la última ecuación ${ }^{4}$.

En el tradicional modelo CRR (Cox, Ross y Rubinstein, 1979) los parámetros para la resolución del sistema de ecuaciones anterior se definen de la siguiente manera:

3 El teorema sostiene que la distribución del promedio de un conjunto de números aleatorios será normal, incluso cuando dichos números al ser considerados en forma individual no se encuentren normalmente distribuidos. Las condiciones por cumplir son: (a) los números aleatorios deben provenir de la misma distribución, (b) deben ser independientes y (c) la distribución debe tener media y varianza finita.

$4 \quad$ Un desarrollo de todas las variantes del modelo binomial se puede encontrar en Van der Hoek y Elliot (2006) y Chance (2007). 


$$
\begin{gathered}
u=e^{\sigma \sqrt{ } \Delta t} \\
d=e^{-\sigma \sqrt{ } \Delta t} \\
p=\frac{1}{2}+\frac{1}{2}\left(\frac{\mu}{\sigma}\right) \sqrt{\Delta t}
\end{gathered}
$$

Los valores para $u, d$ y $p$ son una solución exacta para la ecuación 1 . El modelo CRR establece la cuarta restricción como $u . d=1$, siendo la centralidad un rasgo distintivo. Esto se debe a que el valor proyectado para el subyacente en la etapa 2.dt para el nodo central es igual a su valor inicial ${ }^{5}$. De acuerdo con Whaley (2006), la expresión tradicional correspondiente a las probabilidades de transición (ecuación 3) es:

$$
p=\frac{e^{r \Delta t}-d}{u-d}
$$

Si $\Delta t>\sigma^{2} / \mu^{2}$ el modelo da probabilidades negativas porque: $p=\left(e^{r \Delta t}-e^{(-\sigma / \Delta t)}\right) /\left(e^{(\sigma / \Delta t)}-e^{(-\sigma \vee \Delta t}\right)>1$. El modelo tiene la misma media que el proceso lognormal del subyacente, pero, independientemente del tamaño de $\Delta \mathrm{t}$ la varianza solamente coincide en el límite. Otra propuesta de rejilla binomial es el modelo de Rendleman y Bartter, (1979) y Jarrow y Rudd, (1982). En este modelo se sostiene que la mejor aproximación al valor esperado y media del proceso browniano ocurre si $\mu=r-\left(\sigma^{2} / 2\right)$. Esto implica que las probabilidades de transición tienen valor $p_{u}=p_{d}=1 / 2{ }^{6}$

5 Sustituyendo los valores de $u, d y$ e en la parte izquierda de la varianza (ecuación $2)$, se tiene $\sigma^{2} \Delta t .\left(1-\left(\mu^{2} \Delta t\right) / \sigma^{2}\right)$. Con valores pequeños de $\Delta t$, la segunda ecuación el modelo aproxima a la segunda ecuación del sistema. Para intervalos grandes, cuando $\left(1-1-\left(\mu^{2} \Delta t\right) / \sigma^{2}\right)<0 \mathrm{o} \Delta t>\sigma^{2} / \mu^{2}$, la ecuación de la varianza de la rejilla presenta sesgo negativo (Chance, 2007).

6 La propiedad que las probabilidades neutrales al riesgo deben ser $1 / 2$ se atribuye a Jarrow y Rudd, (1982) 
Los parámetros para resolver el sistema de ecuaciones son: $u=e^{\left(r-\frac{\sigma^{2}}{2}\right) \Delta t+\sigma \sqrt{\Delta t}} ; d=e^{\left(r-\frac{\sigma^{2}}{2}\right) \Delta t-\sigma \sqrt{\Delta t}} ; u . d=e^{2\left(r-\frac{\sigma^{2}}{2}\right) \Delta t}$.

Los parámetros $u$ y $d$ generan una solución para la media y varianza, al ser $p=1 / 2$ y $\mu=r-\left(\sigma^{2} / 2\right)$. Arrojan la misma media y la varianza del proceso lognormal independientemente del tamaño de $\Delta t$, por lo tanto, la rejilla es estable, presenta volatilidad consistente y converge de forma más rápida a la solución analítica que su par CRR (Jarrow y Rudd, 1982 y Jabbour, Kramin y Young, 2001).

\section{Rejillas trinomiales}

Las rejillas trinomiales, comparadas con su par binomial, describen una mayor cantidad de movimientos futuros del subyacente, convergiendo con mayor velocidad en los resultados generados por el modelo continuo, y presentan tres movimientos $(u, m$ y $d$ ) por nodo. Las rejillas trinomiales pueden construirse respetando las premisas utilizadas para su par binomial (Boyle, 1988; Chance, 2007; Derman, Kani y Chriss, 1996; Guthrie, 2009; Hull, 2012; Kamrad y Ritchken, 1991; Tian, 1993; Van der Hoek y Elliot, 2006; Whaley, 2006), así: (a) las probabilidades de transición son positivas en el límite entre 0 y 1 , además su suma debe ser equivalente a la unidad: $p_{u}+p_{m}+p_{d}=1$; (b) la media $(M)$ y la varianza $(V)$ de la distribución trinomial debe ser igual a los primeros dos momentos estocásticos de la distribución lognormal $p_{u} S_{u}+p_{m} S_{m}+p_{d} S_{d}=M$; $p_{u}\left(S^{2} u^{2}-S^{2} M^{2}\right)+p_{m}\left(S^{2} m^{2}-S^{2} M^{2}\right)+p_{d}\left(S^{2} d^{2}-S^{2} M^{2}\right)=S^{2} M^{2} V$ donde $M=e^{r \Delta t}$ y $V=e^{\sigma^{2} \Delta t}-1$.

Conforme fue analizado, el modelo binomial plantea dos parámetros: $u$, $d$ y $m=1$. Boyle, (1988) y Kamrad y Ritchken, (1991) plantean un valor $\lambda$ que regula la amplitud de los movimientos laterales de manera directamente proporcional. El valor de $\lambda$ es directamente proporcional a la amplitud de movimientos, a mayor el valor del coeficiente, mayor será la probabilidad de movimientos laterales, siendo $\lambda \geq 1$. El coeficiente de ascenso tiene la 


$$
M=e^{r \Delta t}
$$

forma funcional $u=e^{\lambda \sigma \nabla \Delta t}, d=e^{\lambda-\sigma / \Delta t} \mathrm{y} m=u$.d. Fijando $u . d=1$ y sujeto a las condiciones de media y varianza, Boyle plantea las probabilidades de transición como

$$
\begin{gathered}
p_{u}=\frac{\left(V+M^{2}-M\right) u-(M-1)}{(u-1)\left(u^{2}-1\right)} \\
p_{d}=\frac{u^{2}\left(V+M^{2}-M\right)-u^{3}(M-1)}{(u-1)\left(u^{2}-1\right)} \\
p_{m}=1-p_{u}-p_{d}
\end{gathered}
$$

Cuando el coeficiente $\lambda$ es igual a 1 , el modelo arroja resultados similares al modelo binomial, ya que la probabilidad de no cambio en el precio es igual a 0 , esto provoca la eliminación de los nodos del medio. Consecuentemente, el coeficiente de ascenso se resume en $u=e^{\lambda \sigma \Delta \Delta t}=e^{\sigma \vee \Delta t}$ y las probabilidades de movimientos ascendentes y descendentes quedan iguales al modelo de Cox, Ross y Rubinstein (1979). Otros planteamientos son los de Tian (1993) y Derman, Kani y Chriss (1996) que asignan valor de $1 / 3$ a las probabilidades de transición.

\section{Rejillas trinomiales y volatilidad cambiante}

Siguiendo el modelo propuesto por Haahtela (2011a), el trabajo con rejillas trinomiales y volatilidades cambiantes para diferentes periodos, requiere fijar la condición de recombinación de los nodos intemedios. Es necesario cumplir la condición expresada en la ecuación (12).

$$
u \cdot d=m^{2}
$$

La centralidad se encuentra asegurada por la condición $m=e^{r \Delta t}$. La lógica del modelo consiste en calcular probabilidades de transición con base en la mayor volatilidad $\left(\sigma_{\max }\right)$. Los movimientos de ascenso y descenso 
correspondientes al subyacente se estiman con la máxima volatilidad, partiendo de las siguientes expresiones:

$$
\begin{gathered}
u=e^{r \Delta t+\sqrt{e^{\left(\lambda \sigma_{\max }\right) 2} \Delta t-1}} \\
d=e^{r \Delta t-\sqrt{e^{\left(\lambda \sigma_{\max }\right) 2} \Delta t-1}} \\
m=e^{r \Delta t}
\end{gathered}
$$

Las probabilidades de transición para el máximo periodo de volatilidad son

$$
\begin{gathered}
p_{u(\max )}=\frac{m^{2}\left(e^{\sigma_{\max }^{2} \Delta t}-1\right)}{u^{2}+m d-u m-u d} \\
p_{d(\max )}=p_{u}\left(\frac{m-u}{d-m}\right) \\
p_{\text {m(max) }}=1-p_{u}-p_{d}
\end{gathered}
$$

Las probabilidades de transición para los periodos de menor volatilidad se derivan de las ecuaciones anteriores, al proporcionar niveles de volatilidad:

$$
\begin{gathered}
p_{u}^{i}=p_{u(\max )}\left(\frac{\sigma_{i}}{\sigma_{\text {max }}}\right)^{2} \\
p_{d}^{i}=p_{d(\max )}\left(\frac{\sigma_{i}}{\sigma_{\max }}\right)^{2} \\
p_{m}^{i}=1-p_{u}^{i}-p_{d}^{i}
\end{gathered}
$$

La mecánica para la construcción de la rejilla es similar a las mencionadas en este trabajo, primero se proyecta el proceso estocástico del subyacente para luego introducir los flujos de pagos de la opción en el 
modelo. El proceso recursivo de valuación de la opción se realiza con las probabilidades de transición para los niveles de volatilidad:

$$
V_{t-1}=\frac{p_{u}^{i} V_{t, u}+p_{m}^{i} V_{t . m}+p_{d}^{i} V_{t, d}}{e^{r t}}
$$

\section{Modelo trinomial desplazado}

En el caso de valorar opciones reales de proyectos del tipo startups ЕвT, con sesgos en la distribución de los valores posibles, una alternativa consiste en adaptar el modelo trinomial partiendo del binomial desplazado (Haahtela, 2011b; Milanesi, Pesce y El Alabi, 2014), en adelante BD. Adicionalmente, dicha alternativa permite aplicar la expresión cuando el subyacente asume valores negativos, en este caso, el proyecto. Asimismo, la forma funcional de la distribución de posibles valores del subyacente es un intermedio entre la normal y lognormal, siendo asimétrica ${ }^{8}$ y, a diferencia de la propuesta de Rubinstein (1983), el parámetro de desplazamiento $(\theta)$ se incorpora fuera del proceso estocástico.

En efecto, la forma de la distribución se descompone en dos partes: (a) probabilística, donde el valor del activo subyacente $\left(V_{\theta}\right)$ sigue un proceso geométrico browniano; (b) en el parámetro de desplazamiento $(\theta)$ determinístico (Camara y Chung, 2006). La ecuación que describe el proceso estocástico del subyacente hasta el horizonte $T$ es

$$
V_{\theta, T}=V_{\theta, 0} e^{\left(\mu-1 / 2 \sigma_{\theta}^{2}\right) T+\sigma \theta \sqrt{T} z}+\theta_{0} e^{\mu t} z \sim N(0,1)
$$

7 Este es una adaptación del proceso difusión desplazado empleado en la valoración de derivados financieros (Rubinstein, 1983).

8 Es una manera intuitiva y flexible de incorporar sesgos potenciales en los valores proyectados de manera determinística o sensibilizando. Se lo puede incorporar en el conjunto de propuestas donde el modelo binomial es transformado para capturar momentos estocásticos de orden superior (Baliero Filho y Rosenfeld, 2004; Milanesi, 2012 y 2014b). 
Donde $V_{\theta}$ es el valor con desplazamiento, la volatilidad desplazada es $\sigma_{\theta}$ y $\theta$ constituye el parámetro de desplazamiento $\left(V_{\theta}, \sigma_{\theta}, \theta\right)$. El valor esperado y el desvío del subyacente en el horizonte $T$ está dado por las siguientes expresiones

$$
\begin{gathered}
V_{\theta t}=\left(V_{\theta, 0}+\theta_{0}\right) e^{\mu t} \\
\sigma_{\theta}\left(V_{t}\right)=\left|V_{\theta, 0}\right| e^{\mu t} \sqrt{e^{\sigma_{\theta}^{2} t}-1}
\end{gathered}
$$

$V_{\theta}$ se expresa en términos absolutos debido a que puede tomar valores negativos o positivos. El insumo principal para la construcción de la rejilla, en este caso trinomial, proviene de la volatilidad del subyancente $\left(\sigma_{\theta}\right)$. Para su estimación se parte de los parámetros $V_{\theta}$ y $\theta$ de la ecuación 24 , por iteración se obtiene $\sigma_{\theta}$, en donde se fija como restricción el valor $\sigma_{\theta}\left(V_{t}\right)$. El valor correspondiente al desvío sesgado $\sigma_{\theta}\left(V_{t}\right)$ se obtiene aplicando análisis de escenarios y simulación. En el caso de volatilidades cambiantes, los valores $\sigma_{\theta} i\left(V_{t}\right)$ corresponden a cada nivel de volatilidad, iterando sobre la ecuación 25 se obtiene los valores para la máxima volatilidad desplazada $\sigma_{\theta} \max \left(V_{t}\right)$ y las volatilidades correspondientes a cada periodo $\sigma_{\theta} i\left(V_{t}\right)$. La máxima volatilidad permite construir los movimientos del subyacente a través de las ecuaciones 12,13 y 14 :

$$
\begin{gathered}
u_{\theta}=e^{r \Delta t+\sqrt{e^{\left(\lambda \sigma_{\theta} \max \left(V_{t}\right)\right)_{2}} \Delta t-1}} \\
d_{\theta}=e^{r \Delta t-\sqrt{e^{\left(\lambda \sigma_{\theta} \max \left(V_{t}\right)\right)_{2}} \Delta t-1}} \\
u_{\theta} \cdot d_{\theta}=m^{2}
\end{gathered}
$$

La centralidad queda asegurada mediante la ecuación 15 y el valor del proyecto para $\Delta t$ para la rejilla trinomial sesgada se estima:

$$
V_{\theta, u, d, m,(t+1)}=\left(u_{\theta} \cdot V_{\theta, t}+\left|\theta_{0}\right| \cdot e^{r \Delta t}\right) ;\left(m \cdot V_{\theta, t}+\left|\theta_{0}\right| \cdot e^{r \Delta t}\right) ;\left(d_{\theta} \cdot V_{\theta, t}+\left|\theta_{0}\right| \cdot e^{r \Delta t}\right)
$$


Donde, $V_{\theta^{\prime} t}$ es el valor desplazado de los activos riesgosos del proyecto, $\theta_{0}$ el valor correspondiente al parámetro de desplazamiento, $r$ el tipo de interés sin riesgo. El movimiento $(u, m \mathrm{y} d)$ es ajustado sumando el valor absoluto del sesgo capitalizado por los periodos $\left(\left|\theta_{0}\right| . e^{r \Delta t}\right)$.

A partir de los movimientos sesgados obtenidos en las ecuaciones 26 , 27 y 28 , y con base en las ecuaciones 16,17 y 18 se construyen las probabilidades de transición desplazadas:

$$
\begin{gathered}
p_{u \theta(\max )}=\frac{m^{2}\left(e^{\sigma \theta_{\max }^{2} \Delta t}-1\right)}{u_{\theta}^{2}+m d_{\theta}-u_{\theta} m-u_{\theta} d_{\theta}} \\
p_{d \theta(\max )}=p_{u \theta}\left(\frac{m-u_{\theta}}{d_{\theta}-m}\right) \\
p_{\operatorname{m\theta }(\max )}=1-p_{u \theta}-p_{d \theta}
\end{gathered}
$$

Luego, las probabilidades intermedias en donde el ajuste es proporcional a las volatilidades segadas:

$$
\begin{gathered}
p_{u \theta}^{i}=p_{u \theta(\max )}\left(\frac{\sigma \theta_{i}}{\sigma \theta_{\text {max }}}\right)^{2} \\
p_{d \theta}^{i}=p_{d \theta(\max )}\left(\frac{\sigma \theta_{i}}{\sigma \theta_{\max }}\right)^{2} \\
p_{m \theta}^{i}=1-p_{u \theta}^{i}-p_{d \theta}^{i}
\end{gathered}
$$

El valor teórico de la opción se obtiene recursivamente:

$$
V_{\theta(t-1)}=\frac{p_{u \Pi}^{i} V_{\theta t, u \theta}+p_{m \Pi}^{i} V_{\theta t . m \theta}+p_{d \Pi}^{i} V_{\theta t, d \theta}}{e^{r t}}
$$




\section{La función de utilidad isoelástica y el modelo trinomial desplazado con volatilidad cambiante}

El uso de funciones de utilidad para valuar los nodos de la rejilla trinomial permite la incorporación del grado de aversión al riesgo del agente. El uso de funciones de utilidad exponenciales del tipo isoelásticas, son herramientas empleadas en rejillas binomiales (Maya Ochoa y Pareja Vasseur, 2014; Milanesi, 2018a y 2019; Pareja Vasseur y Cadavid, 2016). Las funciones de utilidad isoelásticas constituyen un caso especial de la forma hiperbólica de aversión absoluta al riesgo (Merton, 1992) y satisfacen las condiciones de derivada primera positiva y segunda negativa $\left(U^{\prime}(W)>0\right)$; $\left(U^{\prime \prime}(W)<0\right)$. Estas se conocen como CRRA y presentan las siguientes características:

$$
U(W)=\left\{\begin{array}{c}
\frac{W^{\gamma}-1}{1-\gamma} \rightarrow \gamma>0 ; \gamma \neq 1 \\
\log (W) \rightarrow \gamma=1
\end{array}\right.
$$

En la ecuación 36, $\gamma$ representa el nivel de aversión al riesgo, cumpliendo con la condición de Inada ${ }^{9}$, aproximando la utilidad marginal a valores de infinito para riquezas tendientes a cero pero sin permitir utilidad con riqueza igual a cero (Suen, 2009), posibilitando la elasticidad de sustitución intertemporal constante, como condición para asegurar la existencia de equilibrio balanceados (Ljungqvist y Sargent, 2000). La medida de aversión

9 La condición se conoce con el apellido del economista japonés Ken-Ichi Inada, formulada para la función de producción garantizando el crecimiento económico en los modelos neoclásicos de crecimiento. El valor de la función es cero en cero; es diferenciable en todos sus puntos, creciente en $\mathrm{x}$, con derivada decreciente (cóncava), el límite de la derivada cercana al origen es infinito y el límite de la derivada hacia el infinito positivo es cero. La función de utilidad converge a la logarítmica con gamma tendiendo a 1, con la regla de l'Hôpital, donde con $\gamma \rightarrow 1$ numerador y denominador de la función tienden a cero. Al ser diferenciados con respecto a $\gamma$, para tomar el límite de la relación de las derivadas cuando $\gamma \rightarrow 1$, la función de utilidad converge a logarítmica, Maya Ochoa y Pareja Vasseur (2014). Los valores negativos describen una conducta favorable al riesgo, o, en otras palabras, su prima por riesgo es negativa. Cabe destacar que los valores extremos -1 y 1 son los extremos de la función que no explican comportamientos observables. 
al riesgo $(\gamma)$ es crucial en la ecuación 36 y es objeto de inumerables calibraciones producto de investigaciones empíricas ${ }^{10}$. En teoría, $\gamma$ debe fluctuar entre -1 y 1 (Pratt, 1964). El valor que arroja el coeficiente depende de las características del individuo, los valores negativos representan personas favorables al riesgo, los positivos aversos y cero corresponde a personas neutrales al riesgo. En el modelo propuesto, la medida de aversión al riesgo se supondrá variable en el tiempo, a mayores horizontes de tiempo, mayor aversión al riesgo $\left(\gamma_{t}\right)$ producto de una mayor incertidumbre.

En el modelo, el valor de la riqueza $(W)$, es sutituido por el valor proyectado del subyacente $V_{\theta, u, d, m,(t+1)}$, obtenido mediante la ecuación 29. Este es el valor correspondiente al subyacente proyectado con la rejilla trinomial, volatilidad cambiante y sesgo. El grado de aversión al riesgo $\gamma$ se introduce en la función de utilidad (ecuación 35), quedando la expresión siguiente:

$$
U\left(V_{\theta, u, d, m,(t+1)}\right)=\left\{\begin{array}{c}
\frac{V_{\theta, u, d, m,(t+1)} \gamma^{\gamma_{t}}-1}{1-\gamma} \rightarrow \gamma>0 ; \gamma_{t} \neq 1 \\
\log \left(V_{\theta, u, d, m,(t+1)}\right) \rightarrow \gamma_{t}=1
\end{array}\right.
$$

La utilidad esperada $E\left[U\left(V_{\theta, u, d, m,(t+1)}\right)\right]$, se obtiene empleando los coeficientes equivalentes ciertos implícitos sesgados, para cada nivel de probabilidades: ecuaciones 30, 31 y 32, probabilidades de transición máximas; ecuaciones 33, 34 y 35, probabilidades de transición intermedias. Estas son aplicadas recursivamente en cada nodo con la lógica de la ecuación 35 ,

$$
\begin{gathered}
E\left[U\left(V_{\theta, u, d, m,(t+1)}\right)\right] \\
=\left\{\left[p_{u \theta}^{i} U\left(V_{\theta t, u \theta}\right)\right]+\left[p_{m \theta}^{i} U\left(V_{\theta t, m \theta}\right)\right]+\left[p_{d \theta}^{i} U\left(V_{\theta t, d \theta}\right)\right]\right\}
\end{gathered}
$$

La utilidad esperada permite calcular el coeficiente equivalente cierto. Si el agente es neutral al riesgo $(\gamma=0)$, el valor obtenido es el mismo que el arrojado por el modelo trinomial con volatilidades cambiantes,

10 No es objeto en el presente trabajo de analizarlas, estas se pueden encontrar en Pareja Vasseur y Baena (2018) y Chavez, Milanesi y Pesce (2019). 


$$
C E\left(V_{\theta, u, d, m,(t+1)}\right)=\left\{E\left[U\left(V_{\theta, u, d, m,(t+1)}\right)\right] \times\left(1-\gamma_{t}\right)\right\}^{\frac{1}{1-\gamma_{t}}}
$$

El equivalente cierto obtenido se actualiza al tipo sin riesgo con la ecuación siguiente,

$$
C E\left(V_{\theta, u, d, m,(t)}\right)=C E\left(V_{\theta, u, d, m,(t+1)}\right) \cdot e^{-r}
$$

El proceso recursivo brinda una medida monetaria que, incorporada en la función de utilidad, arroja el nivel de utilidad adaptado al coeficiente de aversión al riesgo del agente. Esta surge de la ecuación:

$$
U\left(V_{\theta, u, d, m, t)}\right)=\left\{\begin{array}{c}
\frac{C E\left(V_{\theta, u, d, m, t}\right)^{\gamma_{t}}-1}{1-\gamma} \rightarrow \gamma>0 ; \gamma_{t} \neq 1 \\
\log \left(C E\left(V_{\theta, u, d, m,(t)}\right)\right) \rightarrow \gamma_{t}=1
\end{array}\right.
$$

El modelo trinomial con volatilidad cambiante y aversión al riesgo coincide con los resultados del modelo binomial clásico si: $\bar{\gamma}_{t}=0$ constante en todo periodo de tiempo (neutralidad al riesgo); $\lambda=1$ (equidistancia entre ascenso y descenso simular a binomial), $\sigma_{\theta}$ (volatilidad constante) y $\theta=0$ (inexistencia de sesgo).

\section{El funcionamiento del modelo: análisis de caso}

En este trabajo no se persigue obtener soluciones estadísticas generalizables, por el contrario, se busca estudiar y poner de manifiesto el comportamiento del conjunto de variables que componen el modelo propuesto y sus interacciones, con el fin de justificar su funcionamiento bajo un determinado paradigma del conocimiento, en este caso, los modelos numéricos de valuación de activos reales. Como consecuencia de ello, la metodología seleccionada es el estudio de casos en administración ${ }^{11}$.

11 Debe remarcarse que el estudio de caso, entendido como enfoque metodológico, no debe confundirse con el análisis de caso, entendido como un instrumento de enseñanza. El último busca analizar un aspecto concreto relacionado con las 
El estudio de caso consiste en un proyecto de inversión en I\&D con características distintivas respecto de las inversiones tradicionales, debido al grado de flexibilidad estratégica (opcionalidad) intrínseca generada por las barreras de entrada (Milanesi, 2018b; Rubio Martín y Lamothe Fernández, 2010) ${ }^{12}$. En este tipo de inversiones, el perfil del inversor juega un rol preponderante ${ }^{13}$, pues este tipo de emprendimientos se caracteriza por alta incertidumbre, requerimientos constantes de aportes de capital y desarrollos pautados para horizontes prolongados de tiempo ${ }^{14}$.

organizaciones para fomentar el debate o la discusión, mientras que el primero pretende indagar sobre proposiciones teóricas con el objeto de ampliar y generalizar una teoría (Yin, 1994; Castro Monge, 2010).

12 Presentan dos características objetivas: (a) En los sectores altamente innovadores, los costos de fabricación juegan un rol secundario para definir el precio final del producto, siendo la principal inversión en investigación y desarrollo (I+D), por lo que el costo del capital es uno de los principales factores para explicar el costo del producto; (b) existencia de barreras de entrada como una forma de proteger la innovación, a través de patentes, modelos de utilidad, etc.

13 Fase (a), Inversores aportantes del capital semilla: la figura del inversor suele ser el propio emprendedor (investigador o consorcios como agencias de investigación y universidades), además, mediante el mecanismo de subsidios se obtiene el capital inicial que sirve para financiar los desarrollos de la fase de prototipo y, eventualmente, las primeras etapas empresariales. Fase (b), Inversores ángel y capital de riesgo: en esta, los agentes e inversores institucionales asumen un elevado nivel de riesgo invirtiendo en la etapa inicial a cambio de una participación accionarial. Fase (c), Private equity: caracterizada por inversores institucionales en empresas cerradas maduras y sostenibles en el tiempo; por lo general, la inversión apunta a crear valor en la firma, al detectar un management deficiente o potenciales sinergias en su cartera de negocios; aportan capital o equipo de gerencia.

14 Sus etapas o fases de vida son: (a) Etapa preindustrial: madurada la idea, se determina su viabilidad tanto técnica como empresarial, esta etapa se caracteriza por una fuerte aplicación de recursos y una intensa labor de I+D. (b) Lanzamiento al mercado: en caso de que los resultados de la investigación sean positivos, y tras la protección de la innovación (generalmente mediante patente), se entra en una fase de comercialización. (c) Etapa de crecimiento: caracterizada generalmente por un fuerte incremento de ingresos, hasta que se llega a un punto de equilibrio en el que se satura el mercado. (d) Etapa de madurez: la duración de este período dependerá del tiempo de explotación de la patente y de la introducción de nuevos productos, servicios o tecnologías, que traerán consigo una pérdida de cuota de mercado. 
Se prevé un emprendimiento de base tecnológica, que aún se encuentra en etapa de desarrollo, con un horizonte para la finalización de pruebas y homologación para su lanzamiento en el mercado de 4 periodos $(t 4)$. El valor actual estimado por el método de descuento de flujos de fondos requiere la utilización del enfoque MAD (Marketed Asset Disclaimer), ya que el caso bajo estudio carece de precios observable del riesgo, al no tener activos financieros que repliquen sus flujos de fondos. El valor del proyecto es $V_{0}=-\$ 100000$. Si se considera este valor sin haber analizado las alternativas estratégicas, debe rechazarse la I\&D del proyecto por carecer de valor económico. No obstante, existen alternativas estratégicas que dan sentido $\mathrm{y}$ valor a este tipo de proyectos.

En el caso estudiado, existen dos alternativas ejercibles en $t=4$ : (a) desarrollar la etapa de comercialización del producto, con una inversión estimada de I = \$3000; (b) transferir la licencia, con un ingreso de $\$ 9000$ y costo de transferencia de $\$ 2350000$. La cantidad de pasos para construir la rejilla binomial es de $n=8$, con un $\Delta t=0.5$. La tasa libre de riesgo es del $5 \%$ anual, se supone un sesgo $(\theta)$ en el valor esperado del activo de $|\$ 1000|$. La rejilla trinomial tiene un coeficiente de dispersión entre nodos $(\lambda)$ de 1.5. El coeficiente de aversión al riesgo se supone cambiante y creciente a medida que el horizonte temporal se hace distante, en consonancia con la mayor incertidumbre percibida por el inversor, siendo de $\gamma_{1}=0.4$; $\gamma_{2}=0.56 ; \gamma_{3}=0.7 ; \gamma_{4}=0.8$; un comportamiento claramente adverso al riesgo.

Para estimar la volatilidad desplazada correspondiente a cada periodo se parte de las ecuaciones 24 y 25 . La primera sirve para proyectar el valor sesgado del subyacente mediante escenarios y simulación (Milanesi, Pesce y El Alabi, 2014). Con el valor del desvío estándar sesgado para cada nivel de volatilidad $\sigma_{\theta i}(V t)$ iterando se obtiene el valor porcentual de $\sigma_{\theta i}$ para la máxima volatilidad y el resto de las volatilidades. En la siguiente tabla se exponen las variables de entrada del modelo:

(e) Etapa de declive: caracterizada por un decrecimiento de los ingresos, hasta que el proyecto/empresa no sea sostenible. 
Tabla 1. Variables de entrada del modelo, valor esperado, volatilidad y aversión al riesgo para diferentes horizontes

\begin{tabular}{|c|c|c|c|c|c|}
\hline Periodo & $\mathbf{0}$ & $\mathbf{1}$ & $\mathbf{2}$ & $\mathbf{3}$ & $\mathbf{4}$ \\
\hline$V_{\theta t}=\left(V_{\theta, 0}+\theta_{0}\right) e^{\mu t}$ & $\$ 900.00$ & $\$ 946.14$ & $\$ 994.65$ & $\$ 1045.65$ & $\$ 1099.26$ \\
\hline$\sigma_{\theta} i\left(V_{t}\right)$ & $\$-$ & $\$ 460.00$ & $\$ 582.00$ & $\$ 658.00$ & $\$ 721.00$ \\
\hline$\sigma_{\theta} i$ & & $46.06 \%$ & $38.37 \%$ & $33.35 \%$ & $29.91 \%$ \\
\hline$\gamma$ & 0 & 0.40 & 0.56 & 0.7 & 0.8 \\
\hline
\end{tabular}

Fuente: elaboración propia.

Los valores de la tabla 1 permiten obtener los coeficientes de ascenso, descenso y medio (ecuaciones 26,27 y 28 ), las probabilidades de transcisión desplazadas de máxima volatilidad (ecuaciones 30,31 y 32 ) y las probabilidades de transición intermedias. Estas van proporcionadas según el nivel de volatilidad (ecuaciones 32,33 y 34 ), conforme se expone en la tabla 2 :

Tabla 2. Parámetros rejilla trinomial desplazada con volatilidad cambiante.

\begin{tabular}{|c|c|c|c|c|}
\hline Periodo & $\mathbf{1}$ & $\mathbf{2}$ & $\mathbf{3}$ & $\mathbf{4}$ \\
\hline$u_{\theta}$ & 1.72329055 & 1.72329055 & 1.72329055 & 1.72329055 \\
\hline$m_{\theta}$ & 1.02531512 & 1.02531512 & 1.02531512 & 1.02531512 \\
\hline$d_{\theta}$ & 0.61003706 & 0.61003706 & 0.61003706 & 0.61003706 \\
\hline$p u_{\theta(\max )}$ & 0.1514269 & & & \\
\hline$p m_{\theta(\max )}$ & 0.5940635 & & & \\
\hline$p d_{\theta(\max )}$ & 0.2545096 & & & \\
\hline$p u_{\theta}^{i}$ & & 0.10506498 & 0.07935907 & 0.06383916 \\
\hline$p m_{\theta}^{i}$ & & 0.71834785 & 0.78725879 & 0.82886367 \\
\hline$p d_{\theta}^{i}$ & & 0.17658717 & 0.13338215 & 0.10729717 \\
\hline
\end{tabular}

Fuente: elaboración propia. 
Las probabilidades de transición se ajustan en función al coeficiente $\lambda$, a mayor coeficiente, mayor peso cobra $m$, tendiendo a 1 , mientras que los extremos $(u$ y $d)$ tienden a su par binomial. Para proyectar el recorrido del subyacente se utiliza la ecuación 29 , donde primero se proyecta el valor con sesgo y luego este se extrae. La rejilla queda expuesta de la siguiente manera:

Tabla 3. Valor del proyecto en la rejilla trinomial.

\begin{tabular}{|c|c|c|c|c|c|c|c|c|}
\hline $\mathbf{0}$ & \multicolumn{2}{|c|}{$\mathbf{1}$} & \multicolumn{2}{c|}{$\mathbf{2}$} & \multicolumn{2}{c|}{$\mathbf{3}$} & \multicolumn{2}{c|}{4} \\
\hline$\$-100.00$ & $\$ 525.65$ & $\$ 1621.49$ & $\$ 3528.05$ & $\$ 6832.20$ & $\$ 12545.24$ & $\$ 22410.01$ & $\$ 39429.89$ & $\$ 68780.62$ \\
\hline & $\$-102.53$ & $\$ 538.95$ & $\$ 1662.53$ & $\$ 3617.37$ & $\$ 7005.16$ & $\$ 12862.83$ & $\$ 22977.32$ & $\$ 40428.06$ \\
\hline & $\$-476.28$ & $\$-105.13$ & $\$ 552.60$ & $\$ 1704.62$ & $\$ 3708.94$ & $\$ 7182.49$ & $\$ 13188.45$ & $\$ 23558.99$ \\
\hline & & $\$-488.34$ & $\$-107.79$ & $\$ 566.59$ & $\$ 1747.77$ & $\$ 3802.83$ & $\$ 7364.32$ & $\$ 13522.32$ \\
\hline & & $\$-716.34$ & $\$-500.70$ & $\$-110.52$ & $\$ 580.93$ & $\$ 1792.02$ & $\$ 3899.10$ & $\$ 7550.75$ \\
\hline & & & $\$-734.47$ & $\$-513.38$ & $\$-113.31$ & $\$ 595.64$ & $\$ 1837.38$ & $\$ 3997.81$ \\
\hline & & & $\$-873.56$ & $\$-753.07$ & $\$-526.37$ & $\$-116,18$ & $\$ 610.71$ & $\$ 1883.90$ \\
\hline & & & & $\$-895.68$ & $\$-772.13$ & $\$-539.70$ & $\$-119.12$ & $\$ 626.17$ \\
\hline & & & & $\$-980.53$ & $\$-918.35$ & $\$-791.68$ & $\$-553.36$ & $\$-122.14$ \\
\hline & & & & & $\$-1005.35$ & $\$-941.60$ & $\$-811.72$ & $\$-567.37$ \\
\hline & & & & & $\$-1057.11$ & $\$-1030.80$ & $\$-965.44$ & $\$-832.27$ \\
\hline & & & & & & $\$-1083.87$ & $\$-1056.90$ & $\$-989.88$ \\
\hline & & & & & & $\$-1115.45$ & $\$-1111.31$ & $\$-1083.65$ \\
\hline & & & & & & & $\$-1143.69$ & $\$-1139.44$ \\
\hline & & & & & & & $\$-1162.95$ & $\$-1172.64$ \\
\hline & & & & & & & & $\$-1192.39$ \\
\hline & & & & & & & & $\$-1204.14$ \\
\hline
\end{tabular}

Fuente: elaboración propia.

Luego de esto, en el nodo terminal se debe calcular el valor intrínseco de la opción, para luego aplicar las funciones isoelásticas (ecuación 37) en cada nodo. Después, estimar la utilidad esperada con las probabilidades de transción correspondiente a cada nodo (ecuación 38), el equivalente cierto y su valor actual (ecuaciones 39 y 40). Finalmente, y de manera recursiva, la utilidad correspondiente al equivalente cierto (ecuación 41). A continuación se presenta el proceso: 


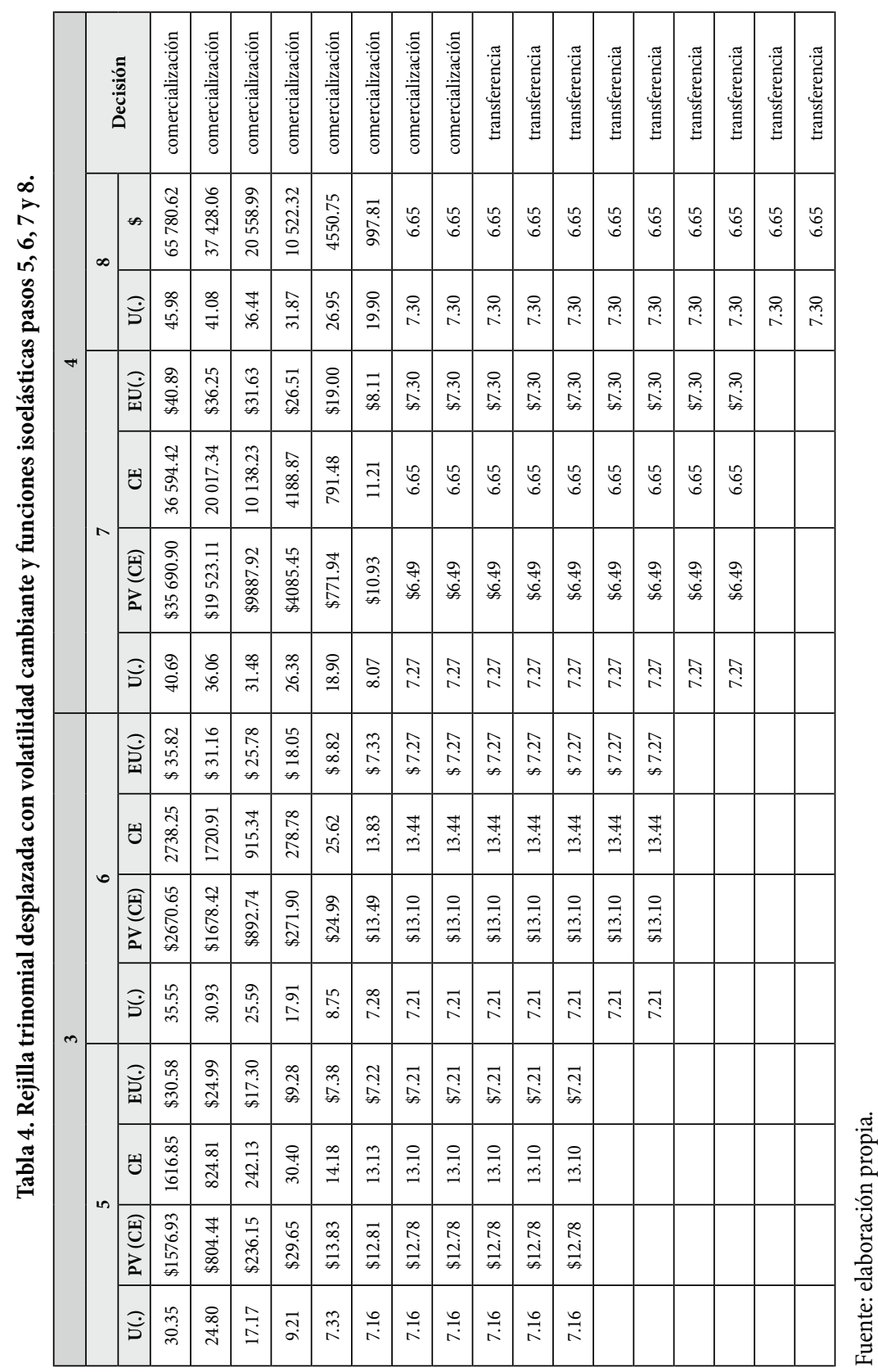


Funciones isoelásticas de utilidad, rejillas trinomiales, | REVISTA ACTIVOS

volatilidad y aversión al riesgo cambiante: un modelo...

\begin{tabular}{|c|c|c|c|c|c|c|c|c|c|c|c|}
\hline \multirow{8}{*}{$N$} & \multirow{4}{*}{ 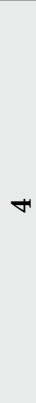 } & & 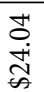 & $\begin{array}{l}\hat{n} \\
\hat{n} \\
6 \\
\sigma\end{array}$ & $\underset{\stackrel{N}{\sigma}}{\stackrel{N}{*}}$ & 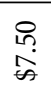 & $\underset{\substack{\infty \\
\infty}}{\stackrel{\infty}{*}}$ & 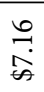 & 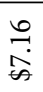 & $\begin{array}{l}0 \\
\stackrel{0}{0} \\
\end{array}$ & $\underset{6}{\stackrel{7}{*}}$ \\
\hline & & પ્પુ & 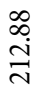 & $\stackrel{\widehat{m}}{\hat{\sigma}}$ & $\underset{\sim}{\stackrel{\sim}{\sim}}$ & $\begin{array}{l}\hat{\text { Dे }} \\
\text { a }\end{array}$ & 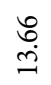 & in & in & in & in \\
\hline & & 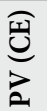 & $\begin{array}{l}\tilde{\sigma} \\
\dot{\vec{d}} \\
\tilde{\sigma}\end{array}$ & $\begin{array}{l}\vec{C} \\
\infty \\
\infty \\
\infty\end{array}$ & $\begin{array}{l}\text { in } \\
\text { in } \\
\text { on }\end{array}$ & $\begin{array}{l}\stackrel{尺}{+} \\
\underset{+\infty}{+}\end{array}$ & $\begin{array}{l}\tilde{m} \\
\stackrel{m}{\omega}\end{array}$ & 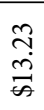 & 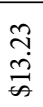 & $\begin{array}{l}\underset{\pi}{n} \\
\underset{\leftrightarrow}{\Leftrightarrow}\end{array}$ & $\begin{array}{l}\underset{\tilde{m}}{m} \\
\stackrel{m}{\Leftrightarrow}\end{array}$ \\
\hline & & $\widehat{\dot{\vec{b}}}$ & 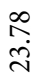 & శ్తి & $\begin{array}{l}\vec{b} \\
\sigma\end{array}$ & $\stackrel{\text { ㄱ. }}{n}$ & $\stackrel{ }{\stackrel{1}{n}}$ & $\stackrel{\infty}{\stackrel{\sim}{\sim}}$ & $\stackrel{\infty}{\stackrel{\Gamma}{r}}$ & $\stackrel{\infty}{\stackrel{\Gamma}{r}}$ & $\stackrel{\infty}{\circ}$ \\
\hline & \multirow{4}{*}{$m$} & 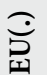 & $\begin{array}{l}\hat{\alpha} \\
\text { nิ }\end{array}$ & में & 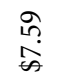 & $\stackrel{m}{\stackrel{n}{n}}$ & 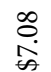 & $\underset{\infty}{\infty}$ & $\stackrel{\substack{0 \\
\infty}}{0}$ & & \\
\hline & & 덩 & $\underset{+}{\stackrel{8}{\infty}}$ & $\begin{array}{l}\infty \\
1 \\
\infty \\
\infty \\
\sim\end{array}$ & $\begin{array}{l}0 \\
\text { in } \\
n\end{array}$ & 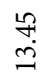 & $\underset{\sim}{\stackrel{H}{\sim}}$ & $\underset{\sim}{\sim}$ & $\underset{\ddot{n}}{\ddot{\sim}}$ & & \\
\hline & & 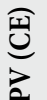 & 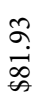 & $\begin{array}{l}\infty \\
\infty \\
\stackrel{\infty}{\infty}\end{array}$ & $\begin{array}{l}\stackrel{7}{n} \\
\stackrel{n}{\infty}\end{array}$ & $\begin{array}{l}\stackrel{7}{\omega} \\
\vec{m}\end{array}$ & $\frac{\widetilde{a}}{\tilde{d}}$ & $\begin{array}{l}\vec{S} \\
\vec{d}\end{array}$ & $\begin{array}{l}\vec{S} \\
\vec{d} \\
\vec{w}\end{array}$ & & \\
\hline & & $\widehat{\dot{\Xi}}$ & 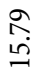 & $\stackrel{\infty}{\infty}$ & $\vec{n}$ & 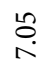 & $\vec{D}$ & $\stackrel{\circ}{\circ}$ & $\stackrel{8}{\circ}$ & & \\
\hline \multirow{8}{*}{-} & \multirow{4}{*}{$\sim$} & ֻॄ & $\begin{array}{l}\vec{I} \\
\dot{0} \\
\dot{H}\end{array}$ & 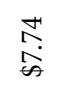 & $\vec{\square}$ & 官. & 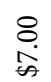 & & & & \\
\hline & & પ્પુ & ָ̊: & $\stackrel{+}{\stackrel{2}{r}}$ & $\exists$ & $\vec{b}$ & 8 & & & & \\
\hline & & $\begin{array}{l}\text { 펄 } \\
\text { 之े }\end{array}$ & $\begin{array}{l}\infty \\
\stackrel{\infty}{\sigma} \\
\stackrel{\theta}{\infty}\end{array}$ & 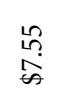 & $\begin{array}{l}\hat{\sigma} \\
\dot{\delta} \\
\infty\end{array}$ & $\begin{array}{l}\underbrace{}_{0} \\
0 \\
0 \\
0\end{array}$ & $\begin{array}{l}\infty \\
0 \\
\infty \\
\infty\end{array}$ & & & & \\
\hline & & ๖े & बे & $\stackrel{\stackrel{\stackrel{n}{n}}{n}}{n}$ & $\hat{\sigma}$ & $\underset{0}{\stackrel{D}{0}}$ & $\underbrace{\infty}_{0}$ & & & & \\
\hline & \multirow{4}{*}{-} & 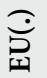 & $\begin{array}{l}\stackrel{0}{a} \\
\text { in }\end{array}$ & 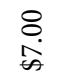 & 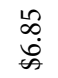 & & & & & & \\
\hline & & 떵 & ঐે & 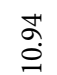 & $\begin{array}{l}\stackrel{n}{n} \\
\stackrel{n}{0}\end{array}$ & & & & & & \\
\hline & & $\begin{array}{l}\text { 평 } \\
\text { : }\end{array}$ & $\begin{array}{l}\widehat{\sigma} \\
\stackrel{\vec{U}}{*}\end{array}$ & $\begin{array}{l}\hat{0} \\
\stackrel{0}{\infty} \\
\dot{\infty}\end{array}$ & $\begin{array}{l}\stackrel{े}{0} \\
\stackrel{\infty}{+}\end{array}$ & & & & & & \\
\hline & & 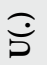 & : & \&్ & $\underset{\substack{n \\
6}}{ }$ & & & & & & \\
\hline
\end{tabular}

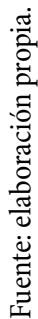


Tabla 6. Valor del proyecto: rejilla trinomial desplazada con volatilidad cambiante $\mathrm{y}$ funciones isoelásticas.

\begin{tabular}{|c|c|c|c|}
\hline \multicolumn{4}{|c|}{ Valor actual } \\
\hline 0 & & & \\
\hline $\mathrm{U}()$. & $\mathrm{PV}(\mathrm{CE})$ & $\mathrm{CE}$ & $\mathrm{EU}()$. \\
\hline 6.83 & $\$ 10.60$ & 10.87 & $\$ 6.97$ \\
\hline
\end{tabular}

Fuente: elaboración propia.

El análisis de sensibilidad expone las variaciones en el valor, en este caso, con modificaciones en el sesgo y en la amplitud de los movimientos correspondientes a la rejilla binomial.

Tabla 7. Análisis de sensibilidad, sesgo y amplitud de movimientos.

\begin{tabular}{|c|c|c|c|c|c|}
\hline$\$ 10,60$ & 0 & -500 & -1000 & -1500 & -2000 \\
\hline 1 & $\$ 10.01$ & $\$ 10.04$ & $\$ 10.42$ & $\$ 11.27$ & $\$ 12.84$ \\
\hline 1,5 & $\$ 9.99$ & $\$ 10.05$ & $\$ 10.60$ & $\$ 10.83$ & $\$ 12.88$ \\
\hline 2 & $\$ 9.98$ & $\$ 10.07$ & $\$ 10.75$ & $\$ 11.20$ & $\$ 11.41$ \\
\hline 2.5 & $\$ 9.98$ & $\$ 10.00$ & $\$ 10.36$ & $\$ 10.44$ & $\$ 13.26$ \\
\hline 3 & $\$ 9.98$ & $\$ 10.06$ & $\$ 10.10$ & $\$ 11.96$ & $\$ 12.51$ \\
\hline
\end{tabular}

Fuente: elaboración propia.

Finalmente, se presentan los diferentes valores, a partir del binomial tradicional, transitando por rejillas trinomiales con volatilidad constante, con y sin sesgo, trinomiales con volatilidad cambiante y trinomiales con volatilidad, sesgo y función isoelástica de utilidad: 
Tabla 8. Valores según parámetros.

\begin{tabular}{|c|c|c|c|c|c|c|}
\hline \multirow{3}{*}{ Parámetros } & Binomial & Trinomial & Trinomial & Trinomial & Trinomial & Trinomial \\
\hline & $\begin{array}{l}\sigma=\mathrm{k} \\
\theta=0\end{array}$ & $\begin{array}{l}\sigma=\mathrm{k} \\
\theta=0\end{array}$ & $\begin{array}{l}\sigma=\mathrm{k} \\
\theta=0\end{array}$ & $\begin{array}{c}\sigma=\mathrm{v} \\
\theta=-1000\end{array}$ & $\begin{array}{c}\sigma=\mathrm{v} \\
\theta=-1000\end{array}$ & $\begin{array}{c}\sigma=\mathrm{v} \\
\theta=-1000\end{array}$ \\
\hline & $\begin{array}{l}\gamma=0 \\
\lambda=1\end{array}$ & $\begin{array}{c}\gamma=0 ; \\
\lambda=1.5\end{array}$ & $\begin{array}{c}\gamma=0 ; \\
\lambda=1.5\end{array}$ & $\begin{array}{c}\gamma=0 ; \\
\lambda=1.5\end{array}$ & $\begin{array}{c}\gamma=0 ; \\
\lambda=1.5\end{array}$ & $\begin{array}{c}\gamma=v ; \\
\lambda=1.5\end{array}$ \\
\hline$\theta$ & 0 & 0 & -1000 & -1000 & -1000 & -1000 \\
\hline$\lambda$ & 1 & 1 & 1 & 1.5 & 1.5 & 1.5 \\
\hline$\sigma$ & $\begin{array}{c}\sigma \\
\text { (constante) }\end{array}$ & $\begin{array}{c}\sigma \\
\text { (constante) }\end{array}$ & $\begin{array}{c}\sigma \\
\text { (constante) }\end{array}$ & $\begin{array}{c}\sigma \\
\text { (constante) }\end{array}$ & $\begin{array}{c}\sigma \\
\text { (variable) }\end{array}$ & $\begin{array}{c}\sigma \\
\text { (variable) }\end{array}$ \\
\hline$\gamma$ & 0 & 0 & 0 & 0 & 0 & $\begin{array}{c}\gamma \\
\text { (variable) }\end{array}$ \\
\hline $\mathrm{U}()$. & $5 . .44$ & 5.44 & 47.35 & 56.79 & 31.96 & 6.83 \\
\hline PV(CE) & 5.44 & 5.44 & 47.35 & 56.79 & 31.96 & 10.6 \\
\hline
\end{tabular}

Fuente: elaboración propia.

En la tabla 8 se puede apreciar el efecto del sesgo, ya que su inexistencia hace que la opción de continuar no sea viable, por ende los valores positivos se encuentran en la transferencia, de tal manera que el binomial y tradicional [\$5.44 / U(5.44)] arrojan resultados similares al asignarse un parámetro $\lambda=1$. Con sesgo, existe posibilidad de continuar y ello es capturado en el resultado [ $\$ 47.35$ / U(47.35)]. Si el parámetro $\lambda>1$, entonces binomial y trinomial no coinciden, arrojando el último un mayor valor dada la distribución en tres nodos [\$56.79/ U(56.79)]. Asimismo, incorporando volatilidad variable, el resultado se ajusta a la baja, producto de los niveles de dispersión menores, quitando peso ponderado a las probabilidades ascendentes [\$31.96 / U(31.96)]. Finalmente, al incorporar aversión al riesgo variable y creciente en el tiempo, el valor se ajusta a la percepción de un sujeto adverso en mercado incompletos, con sesgos y riesgos variables $[\$ 6.83 / \mathrm{U}(10.6)]$. 
Los resultados contenidos en la tabla 8 ponen en evidencia la necesidad de adecuar el modelo a las caracteristicas del proyecto a evaluar y el contexto financiero de referencia. En el caso de proyectos de inversión o emprendimiento tecnológicos, el ciclo de vida producto mercado hace que la volatilidad del emprendimiento sea variable, por eso, el aplicar el modelo de volatilidad variable es una condición necesaria. El mismo razonamiento se aplica para la incorporación de los sesgos en el comportamiento estocástico del subyacente.

Por lo general, las empresas de base tecnológica, su valor y flujos de fondos, no se rigen estrictamente por un supuesto de normalidad. El contexto financiero es un factor crucial, pues, en mercados perfectos, completos y eficientes todos los riesgos del proyecto son considerados de mercado; esto implica que su precio puede replicarse mediante la variabilidad de los flujos de fondos de titulos financieros perfectamente correlacionados, con los ingresos y costos del proyecto.

Sin embargo, tal ideal es dificil de cumplir, más aún en contextos emergentes. En estos casos, un camino está dado por el empleo del enfoque MAD y las funciones isoelasticas de utilidad sensibilizando el coeficiente gamma, que permite replicar riesgos y posiciones frente al riesgo del agente involucrado en la decisión (Smith y Nau, 1995). La necesidad de trabajar con modelos trinomiales esta dada por la mayor especifidad para diagramar el recorrido estocástico (Hull, 2012), y la velocidad para ajustar, en el límite al modelo de Black-Scholes.

\section{Conclusiones}

Frente al no cumplimiento de los supuestos tradicionales relativos a mercados eficientes, perfectos, completos y desarrollados, es menester capturar y valorar tales imperfecciones en el modelo. El modelo binomal y trinomial tradicional con neutralidad al riesgo supone inversores racionales ilimitadamente, capaces de construir carteras réplicas para estimar el riesgo de los flujos de fondos del proyecto, en especial para inversiones estables cuya 
volatilidad se mantenga en determinados rangos. Además, se requiere de un contexto caracterizado pormercados financieros perfectos, eficientes y completos, en donde prácticamente todos los riesgos sean considerados "de mercado" en términos de Smith y Nau (1995).

No obstante, los mercados emergentes, no completos, y en particular, proyectos del tipo I\&D o EBT no ven reflejados sus potenciales riesgos. Es allí donde el modelo debe indagar y brindar herramientas al valuador, que permitan conjugar la ambigüedad del mercado respecto a los datos para estimar riesgo, las características dinámicas de la inversión y las preferencias del inversor. El modelo propuesto toma las rejillas trinomiales como una herramienta que lograr mayor detalle en el mapeo de la granularidad de la rejilla, usa los escenarios y la simulación como técnica para estimar los posibles valores y el sesgo del proyecto; de esta forma se abarcan todos los riesgos del sesgo y se incorporan las funciones isoeláticas de utilidad junto al grado de aversión al riesgo del evaluador.

Esto último es de vital importancia, pues no debe perderese de vista que un proceso de valuación es una actividad intelectual donde el conocimiento del evaluador se transforma en parámetros sencillos para la toma de decisiones, en especial del valor, el cual se transforma en precio perfeccionado en la transacción.

\section{Referencias}

Baliero Filho, R. y Rosenfeld, R. (2004). Testing Option Pricing with Edgeworth Expansion. Physica A: Statistical Mechanis an its Application, 344, 484-490.

Black, F. y Scholes, M. (1972). The Valuation of Options Contracts and a Test of Market Efficiency. Journal of Finance, 27, 399-418.

Black, F. y Scholes, M. (1973). The Pricing of Options and Corporate Liabilities. Journal of Political Economy, 81(3), 637-659.

Boyle, P. (1988). A lattice framework for option pricing with two state variables. Journal of Finance and Quantitative Analysis, 23, 1-12. 
Camara, A y Chung, S. (2006). Option Pricing for the Transformed-Binomial Class. Journal of Futures Markets, 26(8), 759-787.

Chance, D. (2007). A Synthesis of Binomial Option Pricing Models for Lognormally Distributed Assets. https://papers.ssrn.com/sol3/papers.cfm?abstract _id=969834

Chavez, E., Milanesi, G. y Pesce, G. (septiembre de 2019). Estimación de la Aversión al Riesgo Implícita en los precios de mercado de diferentes activos financieros en el mercado argentino. Trabajo presentado en la XIX International Finance Conference organizada por la Universidad Nacional de Córdoba, Argentina.

Copeland, T. y Antikarov, V. (2001). Real Options (1. ${ }^{\mathrm{a}}$ ed.). Texere LLC.

Cox, J., Ross, S. y Rubinstein, M. (1979). Option Pricing: A Simplified Approach. Journal of Financial Economics, 7, 229-263.

Derman, E, Kani, I. y Chriss, N. (febrero del 1996). Implied Trinomial Trees of the Volatility Smile. Quantitative strategies research notes (Goldman-Sachs, ed.).

Guthrie, G. (febrero del 2009). Learning Options and Binomial Trees. Social Science Research Network, 1-19. http://dx.doi.org/10.2139/ssrn.1343430

Haahtela, T. (2011a). Displaced Diffusion Binomial Tree for Real Option Valuation. Social Science Research Network, 1-30. http://dx.doi.org/10.2139/ssrn.1932408

Haahtela, T. (2011b). Recombining trinomial tree for real option valuation with changing volatility. Social Science Research Network, 1-25. http://dx.doi. org/10.2139/ssrn.1932411

Hull, J. (2012). Options, Futures and other Derivatives (Global Edition). Pearson Education Limited (8. ${ }^{\mathrm{a}}$ ed.).

Jabbour, G., Kramin, M. y Young, S. (2001). Two-state Option Pricing: Binomial Models Revisited. Journal of Futures Markets, 21, 987-1001.

Jarrow, R. y Rudd, A. (1982). Aproximate option valuation for arbitrary stochastic processes. Journal of Financial Economics, 10, 347-369.

Kamrad, B. y Ritchken, P. (1991). Multinomial Approximating Models for Options with k State Variables. Management Science, 37(12), 1640-1653.

Ljungqvist, L. y Sargent, T. (2000). Recursive Macroeconomic Theory. MIT Press. Maya Ochoa, C. y Pareja Vasseur, J. (2014). Valoración de opciones a través de equivalentes a certeza. Ecos de Economía, 18(39), 49-72.

Merton, R. (1992). Continuous-Time Finance. Wiley-Blakwell. 
Milanesi, G. (2012). Opciones Reales: el Método Binomial, Asimetría y Curtosis en la Valoración de Empresas de Base Tecnológica. Revista Española de Capital de Riesgo, (2), 41-55.

Milanesi, G. (2014). Modelo Binomial para la Valoración de Empresas y los efectos de la Deuda: Escudo Fiscal y Liquidación de la Firma. Journal of Economics, Finance and Administrative Science, 19(36), 2-10.

Milanesi, G. (2014b). Momentos estocásticos de orden superior y la estimación de la volatidad implícita: aplicación de la expansión de Edgeworth en el modelo de Black-Scholes. Estudios Gerenciales, (30), 336-342.

Milanesi, G. (2018a). Opciones reales y funciones isoelásticas: el caso de la valuación de un proyecto de I\&D en mercados incompletos. Revista Española de Capital de Riesgo, (2), 39-52.

Milanesi, G. (2018b). Un modelo de opciones reales fuzzy y funciones isoelásticas de utilidad para valorar I\&D en mercados incompletos. Estocástica: Administración y Riesgo, 8(2), 205-232.

Milanesi, G. (2019). Valuación de opciones reales, transformación de Edgeworth y funciones isoelásticas de utilidad. Odeon, (16), 123-163.

Milanesi, G, Pesce, G y El Alabi, E. (2014). Valoración de empresas de base tecnológica: Análisis de riesgo y el modelo binomial desplazado. Revista Española de Capital de Riesgo, (4), 15-24.

Pareja Vasseur, J. y Baena J. (2018). Estimación del índice de aversión al riesgo utilizando la función CRRA mediante un diseño experimental. Revista Espacios, 39(13), 29-47.

Pareja Vasseur, J. y Cadavid, C. (2016). Valoración de patentes farmacéuticas a través de opciones reales: equivalentes de certeza y función de utilidad. Contaduria y Administración, (61), 794-814.

Pratt, J. (1964). Risk Aversion in the Small and in the Large. Econometrica, 32(1/2), 122-136.

Rendleman, R. y Bartter, B. (1979). Two-state Option Pricing. Journal of Finance, (34), 1092-1110.

Rubinstein, M. (1983). Displaced Diffusion Option Pricing. Journal of Finance, 38(1), 213-217. 
Rubio Martín, G. y Lamothe Fernández, P. (2010). Valoración de las grandes corporaciones farmacéuticas a través del análisis de sus principales intangibles, con el método de opciones reales. Economía Financiera, 21, 47-74.

Smith, J. (2005). Alternative Approach for Solving Real Options Problems. Decision Analysis, (2), 89-102.

Smith, J. y Nau, R. (1995). Valuing Risky Projects: Option Pricing Theory and Decision Anaysis. Management Science, (5), 795-816.

Suen, R. (2009). Bounding the CRRA Utility Functions. Munich Personal RePec Archive, (13260), 1-16. https://mpra.ub.uni-muenchen.de/13260/1/Bound_ CRRA.pdf

Tian, Y. (1993). A modified lattice approach to option princing. The Journal of Futures Markets, 13(5), 563-577.

Van der Hoek, J. y Elliot, R. (2006). Binomial models in Finance. Springer Science.

Whaley, R. (2006). Derivatives, Markets, Valuation and Risk Management. John Wiley \& Sons.

Wilmott, P. (2009). Frequently Asked Questions in Quantitative Finance (2. $\left.{ }^{\mathrm{a}} \mathrm{ed}.\right)$. John Wiley \& Sons. 